\title{
Testing exoplanet evaporation with multi-transiting systems
}

\author{
James E. Owen^ and Beatriz Campos Estrada \\ Astrophysics Group, Department of Physics, Imperial College London, Prince Consort Rd, London, SW7 2AZ, UK
}

5 December 2019

\begin{abstract}
The photoevaporation model is one of the leading explanations for the evolution of small, close-in planets and the origin of the radius-valley. However, without planet mass measurements, it is challenging to test the photoevaporation scenario. Even if masses are available for individual planets, the host star's unknown EUV/X-ray history makes it difficult to assess the role of photoevaporation. We show that systems with multiple transiting planets are the best in which to rigorously test the photoevaporation model. By scaling one planet to another in a multi-transiting system, the host star's uncertain EUV/X-ray history can be negated. By focusing on systems that contain planets that straddle the radius-valley, one can estimate the minimum-masses of planets above the radius-valley (and thus are assumed to have retained a voluminous hydrogen/helium envelope). This minimum-mass is estimated by assuming that the planet below the radius-valley entirely lost its initial hydrogen/helium envelope, then calculating how massive any planet above the valley needs to be to retain its envelope. We apply this method to 104 planets above the radius gap in 73 systems for which precise enough radii measurements are available. We find excellent agreement with the photoevaporation model. Only two planets (Kepler - 100c \& 142c) appear to be inconsistent, suggesting they had a different formation history or followed a different evolutionary pathway to the bulk of the population. Our method can be used to identify TESS systems that warrant radial-velocity follow-up to further test the photoevaporation model.
\end{abstract}

The software to estimate minimum planet masses is publicly available at: https://github.com/jo276/EvapMass

Key words: planets and satellites: atmospheres - planets and satellites: interiors planets and satellites: physical evolution - planetâĂŞstar interactions

\section{INTRODUCTION}

Recent exoplanet discoveries have fundamentally changed our understanding of what typical planets and planetary systems are. These recent discoveries have been driven by both transit searches, such as the Kepler mission and precision radial-velocity (RV) measurements (e.g. Borucki et al. 2011; Mayor et al. 2011; Thompson et al. 2018). We now know planets with radii in the range $1-4 R_{\oplus}$ and orbital periods $<100$ days are incredibly common (e.g. Howard et al. 2010; Fressin et al. 2013; Silburt et al. 2015; Mulders et al. 2018; Zink et al. 2019), yet the formation of these close-in super-Earths/mini-Neptunes is still poorly understood (e.g. Jankovic et al. 2019). The success of transiting searches for exoplanets means that there are thousands of confirmed ex-

^ E-mail: james.owen@imperial.ac.uk oplanets; yet, the vast majority of these do not possess a mass measurement.

However, the combination of RV follow-up (e.g. Weiss \& Marcy 2014; Marcy et al. 2014) and transit-timing variations (TTVs, e.g. Wu \& Lithwick 2013; Hadden \& Lithwick 2014; Xie 2014; Jontof-Hutter et al. 2016; Hadden \& Lithwick 2017) for a small fraction of transiting exoplanets has allowed some constraints on density and hence composition of exoplanets. Planets with radii $\lesssim 2 \mathrm{R}_{\oplus}$ typically have densities which imply they have a composition similar to Earth (e.g. Dressing et al. 2015; Dorn et al. 2019). Whereas larger planets have lower densities, implying that they must contain a significant fraction of volatiles (e.g. Rogers \& Seager 2010; Weiss \& Marcy 2014; Rogers 2015), in many cases the densities are so low that their densities can only be explained if the planets contain voluminous $\mathrm{H} / \mathrm{He}$ atmospheres (e.g. Jontof-Hutter et al. 2016). With ongoing transit searches ex- 
pected to return thousands more exoplanets (e.g. Günther et al. 2017; Barclay et al. 2018) in the next few years, the fact that the majority of known exoplanets will only have measured radii is likely to remain for the foreseeable future. Therefore, it is crucial that we develop techniques and methods to constrain planetary masses and compositions without direct measurements of planet mass.

Planets with $\mathrm{H} / \mathrm{He}$ atmospheres on short period orbits are liable to mass-loss due to photoevaporation (e.g. Lammer et al. 2003; Murray-Clay et al. 2009; Owen \& Jackson 2012). High-energy irradiation (X-ray and UV) heats up the upper-layers of the $\mathrm{H} / \mathrm{He}$ atmosphere driving a powerful hydrodynamic outflow that causes the atmosphere to lose mass over time (e.g. Baraffe et al. 2005; Lopez et al. 2012; Owen 2019), and in some cases completely removing it. Such massloss has been observed to be occurring through transmission spectroscopy. Originally, this was done using the Lyman- $\alpha$ line (e.g. Vidal-Madjar et al. 2003; Lecavelier Des Etangs et al. 2010; Ehrenreich et al. 2015) and more recently in the $10830 \AA$ HeI line (e.g. Spake et al. 2018; Allart et al. 2018). This idea has led to the hypothesis studied by Owen \& Wu (2013) and Lopez \& Fortney (2013) that the majority of close-in, low-mass planets are born with a composition of a solid core surrounded by a low-mass, but voluminous, $\mathrm{H} / \mathrm{He}$ atmosphere which then experiences photoevaporation and mass-loss. Owen \& Wu (2013) demonstrated that this evolutionary pathway resulted in two distinct planetary structures after billions of years of evolution: firstly, lower mass, more highly irradiated planets typically completely lose their $\mathrm{H} / \mathrm{He}$ atmosphere and finish as a "stripped" core; secondly, higher-mass, less irradiated planets typically end up with a $\mathrm{H} / \mathrm{He}$ atmosphere that consists of $\sim 1 \%$ of the planet's mass, but the radius of the planet is double that of the core. These distinct evolutionary pathways result in a gap in the radius and radius-period distribution of close-in exoplanets and in a distinct prediction of this hypothesis for the origin and evolution of close-in exoplanets, having been confirmed in subsequent theoretical works (e.g. Lopez \& Fortney 2013; Jin et al. 2014; Chen \& Rogers 2016).

A gap has now been observed in the radius distribution, where there is a distinct lack of planets with radii $\sim 1.8 \mathrm{R}_{\oplus}$ (Fulton et al. 2017; Fulton \& Petigura 2018) in agreement with the photoevaporation model. Furthermore, using a set of planets with precise parameters determined through asteroseismology, Van Eylen et al. (2018) demonstrated that this gap is clean, and declines with period in excellent agreement with the photoevaporation model. As argued by Owen \& Wu (2013) and demonstrated in Owen \& Wu (2017), with an observed radius-gap one can use the photoevaporation model to infer the mass-distribution and hence corecomposition of close-in exoplanets. Comparisons to the exoplanet data using the photoevaporation-driven evolution model find that the core composition of these planets is iron-rich and "Earth-like" (Owen \& Wu 2017; Jin \& Mordasini 2018; Wu 2019), providing challenging constraints on the formation of close-in super-Earths and mini-Neptunes.

However, conclusions about the formation pathways and compositions of close-in exoplanets are prefaced on the photoevaporation model being correct and the massloss rates being accurate. There is a degeneracy between the derived core-composition and photoevaporative massloss rates, with lower mass-loss rates favouring lower core densities (Wu 2019; Owen \& Adams 2019). Furthermore, alternative hypothesis have been suggested for the origin of the observed gap in the radius distribution, including corepowered mass-loss (Ginzburg et al. 2018; Gupta \& Schlichting 2019a,b), or two distinct formation pathways for the two sub-groupings. In the latter scenario, the two sub-groupings are water-worlds and rocky, terrestrial planets (Zeng et al. 2019). Recently, it has also been suggested planetesimal impacts may create a similar radius-gap (Wyatt et al. 2019).

The uncertainty over the formation and evolutionary history of close-in exoplanets stems from the fact that the models are under constrained, as the exoplanet mass function remains unknown, but is rather inferred from the chosen evolutionary model. These different models infer different mass functions. In addition, for the photoevaporation model, the majority of the evolution occurs within the first $\sim 100$ Myr of the star's life when its UV \& X-ray output is considerably higher, and the star's current high-energy output is not representative of its earlier history, with at least an order of magnitude spread possible (e.g. Tu et al. 2015). This means that comparisons of individual planets to the photoevaporation models are weakly constraining as one does not know an individual star's high-energy output over it's lifetime.

However, in multi-planet systems while the star's highenergy output is still uncertain we know that all planets in the system experienced the same history. This means multiplanet systems provide an excellent test bed for the photoevaporation model, as has already been demonstrated for the Kepler-36 system (Lopez \& Fortney 2013; Owen \& Morton 2016). In this work, we argue that multi-planet systems which contain planets both above and below the radius-gap (i.e. they "straddle" the gap) are particularly powerful for testing the photoevaporation model. If one adopts the photoevaporation model then the current few billion-year old architecture of the multi-planet systems allows constraints to be placed on the minimum masses of planets above the radius-gap in order to be consistent with the photoevaporation model. Specifically, we ask the question: what is the minimum mass a planet must have in order to retain its $\mathrm{H} / \mathrm{He}$ atmosphere, given another planet in the system entirely lost one?

\section{CONCEPT AND ASSUMPTIONS}

Before we describe our method in detail it is useful to outline the concept of how we can use photoevaporation to constrain planetary masses and the assumptions on which it is based. In order for this method to be applicable we require a multitransiting exoplanet system that contains at least one superEarth that is below the radius gap and at least one miniNeptune with a radius above the radius gap (we use the nomenclature of a super-Earth planet being a planet below the radius-gap and a mini-Neptune being above the radiusgap throughout this work). Although, we caution that the method is not applicable to planets where the mass in the $\mathrm{H} / \mathrm{He}$ atmosphere is comparable to or larger than the mass in the core, wherein self-gravity of the planet's atmosphere becomes important. Therefore, we crudely apply a cut in planetary radii of $<6 R_{\oplus}$.

In order to proceed we then assume that the super- 
Earth planet was born with a $\mathrm{H} / \mathrm{He}$ atmosphere which is then lost. We take the super-Earth planet to have just been able to lose any initial $\mathrm{H} / \mathrm{He}$ atmosphere, hence maximising its mass-loss timescale. Then we solve for the core-mass that equates this maximum mass-loss timescale to the massloss timescale for the mini-Neptune to just retain its current $\mathrm{H} / \mathrm{He}$ atmosphere. This procedure minimises the mass-loss timescale for the mini-Neptune, hence placing a minimum constraint on the core-mass of the mini-Neptune to be consistent with the photoevaporation model. This is because higher core masses will have longer mass-loss timescales and will also be consistent with the photoevaporation models.

\subsection{Assumptions}

The assumptions are based on the general conclusions obtained by Owen \& Wu (2017), Jin \& Mordasini (2018) and $\mathrm{Wu}(2019)$ when applying the photoevaporation model to the exoplanet radius data alone. The assumptions are as follows:

(i) We assume that the core-composition of all planets in the multi-transiting systems are identical.

(ii) We assume that all planets have remained on the currently observed orbits since disc dispersal.

(iii) We assume that all planets accreted a $\mathrm{H} / \mathrm{He}$ atmosphere from the protoplanetary disc that initially contained an atmospheric mass $\gtrsim 1 \%$.

(iv) Finally, in addition to the first assumption, we further assume that the composition of the cores is "Earth-like" containing $1 / 3$ iron and $2 / 3$ silicate rocks by mass.

It is important to emphasise that none of these assumptions can be convincingly argued from a first-principled approach to planet formation. Rather these assumptions have been inferred, by comparing the photoevaporation model to the exoplanet data. Now any planet that has a measured mass below that required to be consistent with the photoevaporation model is likely to result from the breaking of one of the above assumptions. Such a comparison would allow the identification of planetary systems that could have undergone giant impacts after the disc disperses (e.g. Inamdar \& Schlichting 2016), or have ice-rich cores, or those with variable core compositions (e.g. Raymond et al. 2018). Finally, if the measured masses are typically below those required to be consistent with the photoevaporation model, then the model can be ruled out as the origin of the observed radius gap.

\subsection{Basic Expectations}

Before we proceed with numerical solutions, we can get a basic expectation of how the derived minimum mass depends on the parameters of the multi-planet system using the analytic scalings of Owen \& Wu (2017). We adopt the energy-limited mass-loss formula, $\dot{m}=\eta \pi R_{p}^{3} L_{\mathrm{HE}} / 4 \pi a^{2} G M_{p}$, with $\eta$ being the mass-loss efficiency, $R_{p}, M_{p}$ and $a$ the planet's mass, radius and separation, $L_{\mathrm{HE}}$ the high energy luminosity of the star during its active period and $G$ the gravitational constant. In this sub-section, we take the maximum mass-loss timescale for the planet's to occur for an envelope mass fraction $X_{2}$ that doubles the core's radius, i.e. $R_{p}=2 R_{c}$, with $R_{c}$ the core's radius. Therefore, writing the mass-loss timescale $t_{\dot{m}} \equiv M_{\mathrm{atm}} / \dot{m}$, we find the maximum mass-loss timescale is approximately:

$t_{\dot{m}}^{\max } \approx \frac{a^{2} G M_{p}^{2} X_{2}}{\eta 2 R_{c}^{3} L_{H E}}$

We now take the above expression to represent the maximum mass-loss timescale for the super-Earth. We can now say the mass-loss timescale for the mini-Neptune must be larger than this in order to remain gaseous. Crudely, also evaluating this at the point of maximum mass-loss timescale for the mini-Neptune (i.e. when the atmosphere mass fraction is $X_{2}$ ), we can then find a constraint on the minimum mass of the mini-Neptune. This gives:

$$
\begin{aligned}
t_{\dot{m}}^{\text {gas }} & \geq t_{\dot{m}}^{\text {rock }} \\
\frac{a_{g}^{2} M_{g}^{2}}{\eta_{g} R_{c, g}^{3}} & \geq \frac{a_{R}^{2} M_{R}^{2}}{\eta_{R} R_{c, R}^{3}} \\
M_{g} & \geq M_{R}\left(\frac{a_{R}}{a_{g}}\right)\left(\frac{\eta_{g}}{\eta_{R}}\right)^{1 / 2}\left(\frac{R_{c, g}}{R_{c, R}}\right)^{3 / 2}
\end{aligned}
$$

where the sub-script $R$ refers to the "rocky" super-Earth and $g$ refers to the "gaseous" mini-Neptune. Since both massloss timescales depend inversely on $L_{H E}$ this minimum mass is independent of the unknown high-energy flux history of the star. Now adopting the simple mass-radius relation for the solid cores $\left(M_{c} \propto R_{c}^{4}\right.$, e.g., Valencia et al. 2007), we can further simplify Equation 2 to become:

$$
\begin{aligned}
& M_{g} \geq M_{R}\left(\frac{a_{R}}{a_{g}}\right)^{8 / 5}\left(\frac{\eta_{g}}{\eta_{R}}\right)^{4 / 5} \\
& M_{g} \geq 5.1 M_{\oplus}\left(\frac{R_{R}}{1.5 \mathrm{R}_{\oplus}}\right)^{4}\left(\frac{a_{R}}{a_{g}}\right)^{8 / 5}\left(\frac{\eta_{g}}{\eta_{R}}\right)^{4 / 5}
\end{aligned}
$$

were the last inequality has been evaluated for Earth-like composition cores, in terms of the observable radius of the planet below the radius gap. Now finally, if one adopts the scaling of the mass-loss efficiency with escape velocity as $\eta \propto v_{\mathrm{esc}}^{-2}$ from Owen \& Wu (2017), we find a simple relation between the minimum mass of the gaseous planet and the ratio of the orbital separations as:

$M_{g} \geq 5.1 M_{\oplus}\left(\frac{R_{R}}{1.5 \mathrm{R}_{\oplus}}\right)^{4}\left(\frac{a_{R}}{a_{g}}\right)$

Unsurprisingly, one can see the most constraining systems for the photoevaporation model are those that contain a large super-Earth, or those that contain a super-Earth that is exterior to the mini-Neptune. But, those multi-planet systems that contain a small super-Earth interior to a miniNeptune are unlikely to be strongly constraining.

As a simple demonstration we can apply the above model to the Kepler-36 system (Carter et al. 2012), which contains two planets which straddle the gap. Planet $\mathrm{b}$ has a radius of $1.49 \mathrm{R}_{\oplus}$ and a separation of $0.1153 \mathrm{AU}$ while planet c has a radius of $3.68 \mathrm{R}_{\oplus}$ and a separation of $0.1283 \mathrm{AU}$ (Carter et al. 2012). Therefore, Equation 4 implies in order to be consistent with the photoevaporation model planet $\mathrm{c}$ should have a mass $\gtrsim 4.6 \mathrm{M}_{\oplus}$. This minimum mass is consistent with its measured mass of $\sim 8.1 \mathrm{M}_{\oplus}$ (Carter et al. 2012), implying the current observed architecture of the Kepler36 system is consistent with the photoevaporation scenario. This result is not surprising given detailed studies of the 
Kepler-36 system have already shown it to be in agreement with the photoevaporation scenario (e.g. Lopez \& Fortney 2013; Owen \& Morton 2016).

\section{OVERVIEW OF THE METHOD}

While the discussion and derivation in Section 2.2 was a useful prelude to demonstrate the idea, the resulting Equation 4 neglected several key aspects that need to be included before we can robustly compare the photoevaporation model to real systems.

Specifically, we assumed that the envelope-mass fraction at which the mass-loss timescale was maximised is independent of planetary properties. Furthermore, we took the gaseous planet to also have an envelope mass-fraction which maximises its mass-loss timescale. Such an assumption while useful, and true on average, is not true for specific planets which may have envelope mass fractions larger than that required to maximise the mass-loss timescale. An envelope-mass fraction larger than this will result in a shorter envelope-mass fraction and require a larger planet mass to compensate. We also need to account for the fact that planet's $\mathrm{H} / \mathrm{He}$ atmospheres contract over time and were larger when mass-loss was important compared to when we observe them today. Finally, we also need to account for observational errors in the planetary properties.

However, the basic procedure remains the same. We are solving for the mass-loss timescale of the gaseous planet such that it is greater than or equal to the maximum mass-loss timescale the rocky planet would have had for any atmosphere mass-fraction (provided it's $<1$ ).

Therefore, the first goal is to find the maximum massloss timescale for the now supposed stripped core and the envelope mass-fraction that maximises it. Namely, we are maximising the ratio:

$$
\frac{X\left(M_{p}, a\right)}{\eta\left(R_{p}, M_{p}, a\right) R_{p}\left(X, M_{p}, a\right)^{3}}
$$

with $X$ now the general envelope mass fraction $X \equiv$ $M_{\text {env }} / M_{\text {core }}$. We assume that $M_{p}=M_{c}$ and $M_{c}$ is obtained from a mass-radius relation for the solid core, for which we use the Fortney et al. (2007) relations. In this work we have chosen to adopt the simple mass-loss efficiency of Owen \& Wu (2017). However, the model can equally be applied to any mass-loss model, for example detailed numerical radiation-hydrodynamic simulations (e.g. Owen \& Jackson 2012; Kubyshkina et al. 2018). In-fact finding planetary systems that are inconsistent with our test may point, not to errors in the underlying photoevaporation scenario, but rather errors in the mass-loss efficiency.

\subsection{Envelope Structure Model}

In order to convert the envelope mass fraction into a planetary radius or vice-versa we need an envelope structure model. We use the simple semi-analytic envelope structure model adopted by Owen \& Wu (2017) where the envelope consists of an adiabatic interior (with adiabatic index $\gamma$ ) from the surface of the core to a radiative-convective boundary at radius $R_{\text {rcb }}$ and density $\rho_{\text {rcb }}$, followed by an isothermal radiative layer at the equilibrium temperature $\left(T_{\mathrm{eq}}\right)$ reaching the photosphere at the planet's radius ${ }^{1}$. We choose this semi-analytic approach rather than solving for the exact structure (e.g. by using MESA, Paxton et al. 2011, 2013) as it is computationally inexpensive, this allows us to include errors in the planetary parameters by Monte-Carlo sampling (Section 3.3) and quickly analyse a large number of planetary systems.

The density profile in the convective interior is adiabatic and can be approximated by $^{2}$ :

$\rho \approx \rho_{\mathrm{rcb}}\left[\nabla_{\mathrm{ab}}\left(\frac{G M_{c}}{c_{s}^{2} R_{\mathrm{rcb}}}\right)\left(\frac{R_{\mathrm{rcb}}}{r}-1\right)\right]^{1 /(\gamma-1)}$

where $\nabla_{\mathrm{ab}}$ is the adiabatic gradient, $c_{s}$ is the isothermal sound-speed, and $r$ is the radius from the centre of the core. This allows the mass in the convective interior to be written as:

$M_{\mathrm{env}} \approx 4 \pi R_{\mathrm{rcb}}^{3} \rho_{\mathrm{rcb}}\left(\nabla_{\mathrm{ab}} \frac{G M_{c}}{c_{S}^{2} R_{\mathrm{rcb}}}\right)^{1 /(\gamma-1)} I_{2}\left(R_{c} / R_{\mathrm{rcb}}, \gamma\right)$

where $I_{n}$ is a dimensionless integral of the form:

$I_{n}\left(R_{c} / R_{\mathrm{rcb}}, \gamma\right)=\int_{R c / R_{\mathrm{rcb}}}^{1} x^{n}\left(x^{-1}-1\right)^{1 /(\gamma-1)} \mathrm{d} x$

The density profile in the isothermal radiative layer can be approximated by:

$\rho=\rho_{\mathrm{rcb}} \exp \left(-\frac{R-R_{\mathrm{rcb}}}{H}\right)$

with $H=c_{S}^{2} R_{\mathrm{rcb}}^{2} / G M_{p}$ the isothermal scale height. Note, Equation 9 assumes the isothermal radiative atmosphere is thin, and the gravitational acceleration is constant. Additionally, in most cases we assume that the envelope mass is entirely contained within the convective interior and the isothermal radiative layer does not contribute to the envelope mass. Finally, in order to evaluate the density at the radiative convective boundary we equate the temperature gradient across the radiative-convective boundary. Owen \& $\mathrm{Wu}(2017)$ demonstrate that for an opacity law of the form $\kappa=\kappa_{0} P^{\alpha} T^{\beta}$ this gives the density at the radiative convective boundary of:

$\rho_{\mathrm{rcb}} \approx\left(\frac{m u}{k_{b}}\right)\left[\left(\frac{I_{2}}{I_{1}}\right) \frac{64 \pi \sigma T_{\mathrm{eq}}^{3-\alpha-\beta} R_{\mathrm{rcb}} \tau_{\mathrm{KH}}}{3 \kappa_{0} M_{p} X}\right]^{1 /(1+\alpha)}$

with $\tau_{\mathrm{KH}}$ the Kelvin-Helmholtz timescale of the convective interior, which we equate to the age of the planet, and $\sigma$ the Stefan-Boltzmann constant. Following, Owen \& Wu (2017) and $\mathrm{Wu}(2019)$ we select $\gamma=5 / 3, \alpha=0.68, \beta=0.45$ and finally, $\kappa_{0}=4.79 \times 10^{-8}$ when pressure and temperature are expressed in cgs units.

\subsection{Calculation of the minimum planetary mass}

The dependence of the planetary structure on age (i.e. younger planets have lower mass envelopes compared to

1 It is of course an assumption that the interior is convective; however, at this stage such a choice is the natural starting point. 2 This assumes that the mass contained in the envelope near the radiative convective boundary is negligible, this approximation breaks down when the radiative convective boundary is thin. 


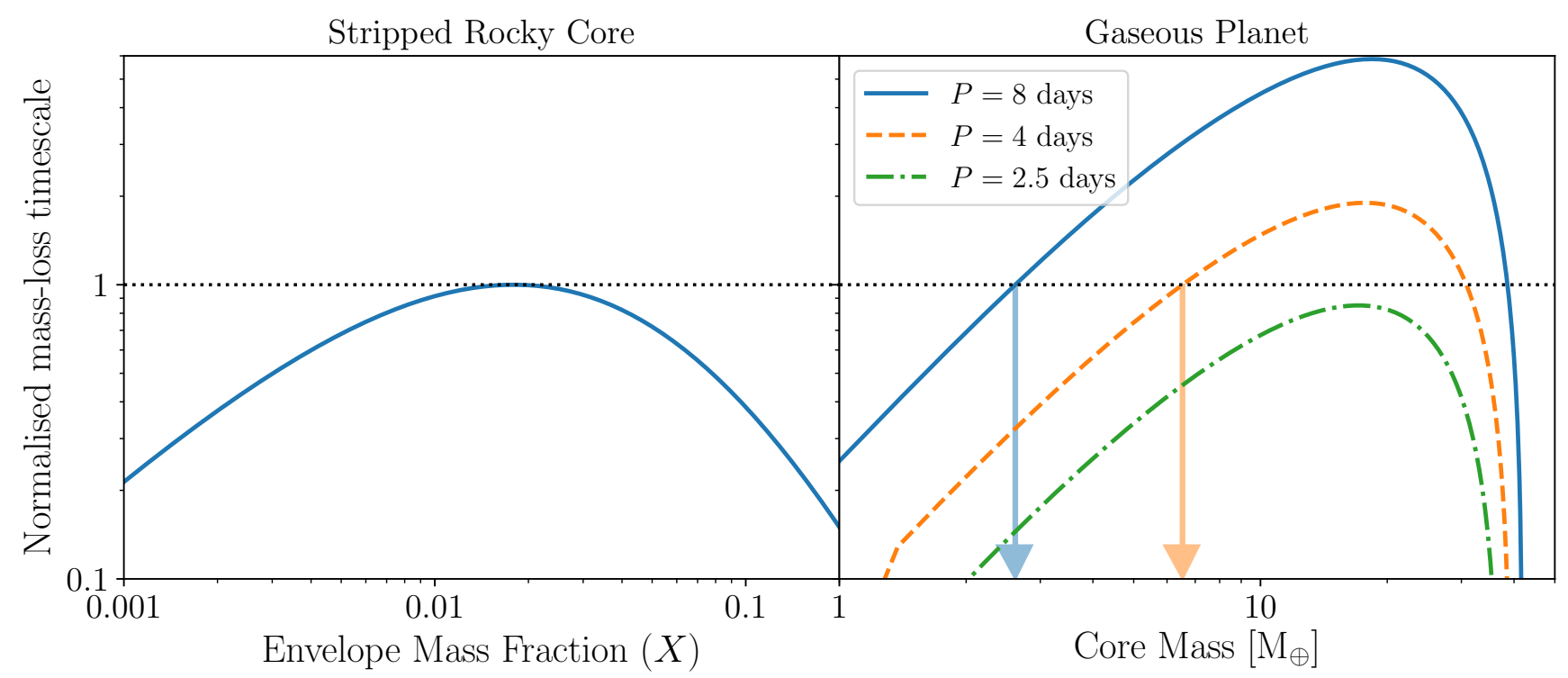

Figure 1. The left panel shows the mass-loss timescale as a function of envelope mass fraction for a $1.5 \mathrm{R}_{\oplus}$ planet with a period of 5 days, which we assume has been striped by photoevaporation. The mass-loss timescale is normalised to its maximum. The right panel shows the mass-loss timescale as a function of core-mass for a $2.5 \mathrm{R}_{\oplus}$ planet, which we assume has retained a $\mathrm{H} / \mathrm{He}$ envelope at periods of 2.5, 4 and 8 days. By equating the maximum mass-loss timescale for the assumed striped rocky core to the assumed gaseous planet we can find the minimum core mass of the gaseous planet to be consistent with the photoevaporation scenario (shown by the arrows). We find a minimum mass of $2.62 \mathrm{M}_{\oplus}$ if the gaseous planet had an orbital period of 8 days and $6.53 \mathrm{M}_{\oplus}$ if it had an orbital period of 4 days and no solution consistent with the photoevaporation model if it had an orbital period of 2.5 days.

the older planets with the same radius), means that we cannot quite ignore the age at which mass-loss is important and the current age of the planets. Although, as we show in Section 4 this trend is weak. Therefore, we select two timescales, $t_{\text {young }}=100 \mathrm{Myr}$, which is when we equate the two mass-loss timescales and $t_{\text {old }}=$ system age, which is when we compare to the observed radius of the supposedly gaseous planet. Therefore, when we find the maximum massloss timescale for the rocky planet in maximising Equation 5 we set $\tau_{\mathrm{KH}}=t_{\text {young }}$ and we also equate this to the mass-loss timescale for the gaseous planet's atmosphere; however, we also constrain the gaseous planet's atmosphere to have an envelope mass-fraction consistent with the observed planet's radius at $t_{\text {old }}$. This means the planetary radius that was adopted for the gaseous planet when it's mass-loss timescale is equated to the maximum mass-loss timescale for the rocky planet is slightly larger than the observed radius.

\subsubsection{Demonstration Systems}

Before we apply our method to real observed planetary systems it is useful to demonstrate the approach for representative systems. As the method of equating the mass-loss timescales typically results in either two solutions for the mass of the gaseous planet or no solution, both outcomes are easy to understand.

We consider a two planets system consisting of a $1.5 \mathrm{R}_{\oplus}$ planet (planet b) with an orbital period of 5 days and a second planet with a radius of $2.5 \mathrm{R}_{\oplus}$ (planet c) whose period we vary. We take planet $\mathrm{b}$ as a stripped rocky core (the super-Earth) and planet $\mathrm{c}$ as a planet that retained a $\mathrm{H} / \mathrm{He}$ atmosphere (the mini-Neptune). Therefore, we wish to max- imise the mass-loss timescale of planet $b$, assuming it had an atmosphere, this is shown in the left-hand panel of Figure 1, where we plot the mass-loss timescale as a function of envelope mass fraction for planet $\mathrm{b}$. This mass-loss timescale clearly peaks at an envelope mass-fraction of a few percent as expected. Next, for planet c we compute its mass-loss timescale as a function of core mass, requiring its radius to be $2.5 \mathrm{R}_{\oplus}$. Planet c's mass-loss timescale is shown in the right panel of Figure 1. This mass-loss timescale is short at low core masses because any atmosphere is weakly bound to the core. It is also short at high core masses because the envelope mass fraction is small (i.e. as the core's radius approaches the planet's radius the envelope mass fraction approaches zero and the mass-loss timescale also tends to zero).

Thus, when equating the maximum mass-loss timescale for planet $\mathrm{b}$ to planet $\mathrm{c}$ (see the right-panel of Figure 1), we can clearly see that there is either two solutions, or no solutions. The two solutions are either a lower core mass with a substantial $(X \gtrsim 1 \%)$ envelope mass fraction or at high core mass with very little envelope $(X \ll 1 \%)$. Now clearly, if planet $\mathrm{c}$ has a period of 2.5 days, all core-masses have a mass-loss timescale less than the maximum of planet $b$ then there is no solution that is consistent with planet $b$ having been completely stripped of a substantial envelope and planet $\mathrm{c}$ retaining one.

Since the inference from the photoevaporation model, when compared to the radius data is that planets are born with a substantial atmosphere and we are interested in a minimum mass to be consistent with the photoevaporation model we select the lower mass solution, as shown by the arrows in the right-panel of Figure 1, with closer in gaseous 


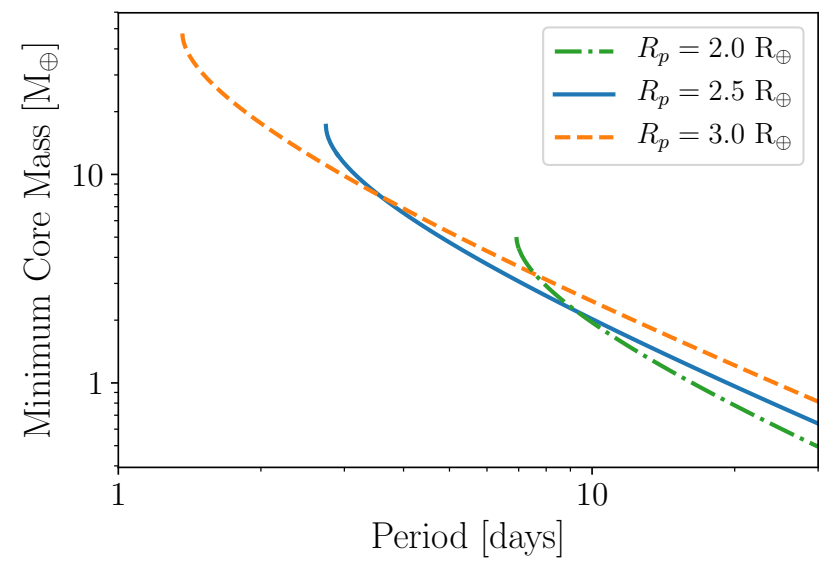

Figure 2. The minimum core mass to be consistent with photoevaporation for a mini-Neptune as a function of orbital period with a super-Earth in the same system that has a radius of $1.5 \mathrm{R}_{\oplus}$ and period of 5 days. This minimum core mass is shown for a miniNeptune with radii of $2.0,2.5 \& 3.0 \mathrm{R}_{\oplus}$. At long periods note the approximate $M_{c} \propto P^{-2 / 3}$ power-law, as expected from Equation 4 .

planets requiring higher core masses, as expected from our discussion in Section 2.2.

In our numerical method to ensure we find the correct solution we maximise the mass-loss timescale for the gaseous planet and then use a bounded root finder to search for a solution between the maximised core mass and a lower bound. In our analysis we set this lower bound to $0.1 \mathrm{M}_{\oplus}$. Therefore, any mini-Neptune whose mass-loss timescale is larger than the super-Earth for $0.1 \mathrm{M}_{\oplus}$ are just assigned a minimum mass of $<0.1 \mathrm{M}_{\oplus}$.

We can assess how our minimum mass estimate for planet $\mathrm{c}$ would vary if we change its radius. This is shown for planetary radii where we plot the minimum core mass as a function of period for planet $\mathrm{c}$ with a radius of 2.0, 2.5 \& $3.0 \mathrm{R}_{\oplus}$ in Figure 2. This shows, as expected, in general a larger planet, which one would expect to have a larger envelope mass-fraction requires a higher minimum core mass. However, larger planets can also be consistent closer to the star, where the larger planetary radii permit larger cores masses to fit inside that radius. For example, the $\gtrsim 20 \mathrm{M}_{\oplus}$ minimum core masses that appear for the $3.0 \mathrm{R}_{\oplus}$ planet at short periods are not possible for the $2.0 R_{\oplus}$ planet, since a $\sim 20 \mathrm{M}_{\oplus}$ core is larger than $2.0 \mathrm{R}_{\oplus}$.

Finally, in Figure 3 we assess how our choice of the age at which to evaluate the planetary structure affects our results. Here we plot the minimum core mass for planet $\mathrm{c}$ (with a radius of $2.5 \mathrm{R}_{\oplus}$ ) as a function of period for choices of $t_{K H}$ of 50,100 (our default choice) and 200 Myr. This shows, that the minimum estimated core masses do not strongly depend on this choice and such differences are likely to be small compared to those caused by errors in the planetary parameters (see Section 3.3).

\subsection{Inclusion of errors on planetary parameters}

All of the key input parameters contain measurement errors and this must be folded into our analysis. Firstly, we must check that the multi-planet system robustly straddles

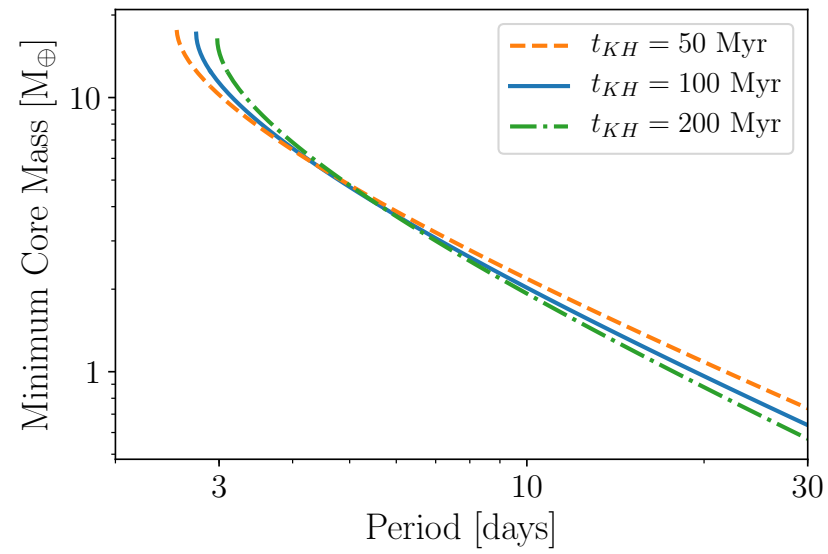

Figure 3. The minimum core mass to be consistent with photoevaporation for a mini-Neptune with a radius of $2.5 \mathrm{R}_{\oplus}$ as a function of orbital period with a super-Earth in the same system that has a radius of $1.5 \mathrm{R}_{\oplus}$ and period of 5 days. This minimum core mass is shown for three choices of the young planet's KelvinHelmholtz timescales ( $t_{\text {young }}$ ) of 50, 100, \& 200 Myr. At long periods note the approximate $M_{c} \propto P^{-2 / 3}$ power-law, as expected from Equation 4.

the radius-gap. We do this by taking that the super-Earth's radius is below the gap to $2 \sigma$ and that the mini-Neptune's radius is above the gap to $2 \sigma$.

In order to include errors on the minimum mass of the gaseous planet we assume Gaussian errors on all the planetary and stellar parameters that are independent ${ }^{3}$. We then randomly draw from each of the planetary and stellar parameters and perform our minimum-mass estimate, we do this Monte Carlo sampling 3000 times and the minimum mass is then given as a $95 \%$ upper-limit unless otherwise stated.

\section{RESULTS}

Here we apply our method to observed exoplanet multitransiting systems and then compare our estimated minimum masses to those systems that have constraints on their masses.

\subsection{Kepler-36}

We return again to the photoevaporative benchmark system Kepler-36 and apply our full method to the observed system. We take the observed stellar and planetary parameters including errors from Carter et al. (2012). Following the discussion in Section 3.3 we randomly sample the stellar and planetary parameters and calculate the minimum mass for each random draw. The resulting distribution for the minimum planetary mass of Kepler-36 is shown in Figure 4 which yields a $95 \%$ upper-mass limit for the system to be consistent with photoevaporation of $4.99 \mathrm{M}_{\oplus}$, similar to the

${ }^{3}$ In reality one could use planetary parameters provided by MCMC chains from the transit fitting procedure, that include any co-variances between the planetary parameters. 


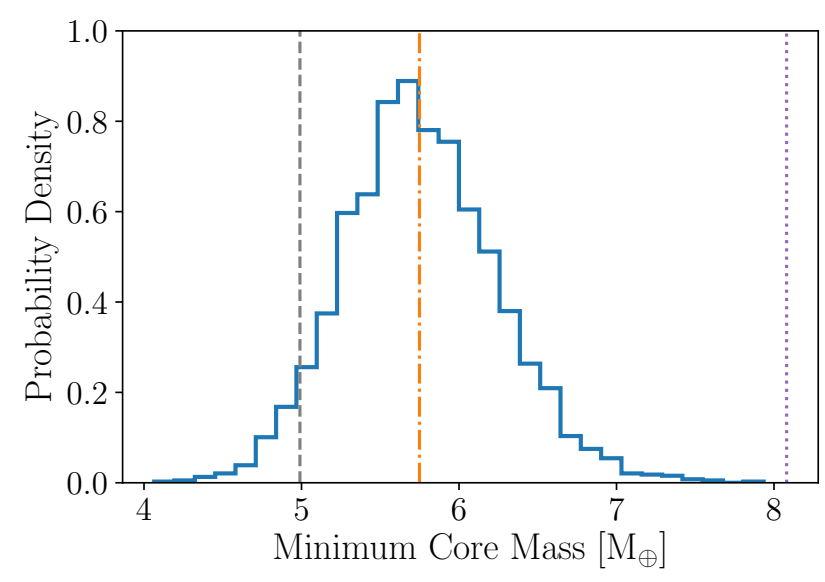

Figure 4. The distribution of minimum core-masses found for Kepler-36c. The dashed line gives the $95 \%$ upper limit of $4.99 \mathrm{M}_{\oplus}$, the dot-dashed line gives the value if the evaluation just used the best fitting parameters for the Kepler-36 system and the dotted line shows best-fit measured mass for Kepler-36c from Carter et al. (2012).

$\sim 4.6 \mathrm{M}_{\oplus}$ upper limit we obtained from simple arguments in Section 2.2, and as expected is consistent with its measured mass, indicating that the Kepler-36 system is consistent with the photoevaporative scenario and the assumptions listed in Section 2.1.

By artificially setting the errors on the stellar and planetary parameters to zero in turn, we find by far the most dominant source of spread in the minimum mass estimates is driven by the radius of the super-Earth Kepler-36b. With the radius of Kepler-36b fixed to its best-fit value the $95 \%$ upper limit for the minimum mass of Kepler-36c is now $\sim 5.5 \mathrm{M}_{\oplus}$ only slightly smaller than the value obtained by just evaluating the best fit for all system parameters. This is not surprising, as the inferred mass of the rocky planet depends strongly (roughly $\propto R_{p}^{4}$ ) on its radius and the minimum mass for the gaseous planet scales linearly with the mass of the rocky one (Section 2.2). Therefore, even small radius errors (which are $2.3 \%$ for Kepler-36b) on the super-Earth planet dominate the precision on the minimum mass estimate.

\subsection{Asteroseismic sample}

Here we work with the asteroseismic sample of Van Eylen et al. (2018), this set contains 24 multi-planet systems of which 13 straddle the radius-gap (for analysis in this subsection we use the radius-gap as a function of period defined by Van Eylen et al. 2018 - we use their expression for the position determined using support vector machines - i.e. the line shown in their Figure 7), 12 of which contain miniNeptunes with radii $<6 R_{\oplus}$. These systems are shown in Figure 5. Eight of these systems have some published mass constraint (including upper-limits) ${ }^{4}$. In total we compute mass estimates for 16 mini-Neptunes. The estimated minimum masses at the $95 \%$ upper-limit level for these systems along

4 As listed in NASA's exoplanet archive, in the case of multiple masses listed we take the most recent values without prejudice.

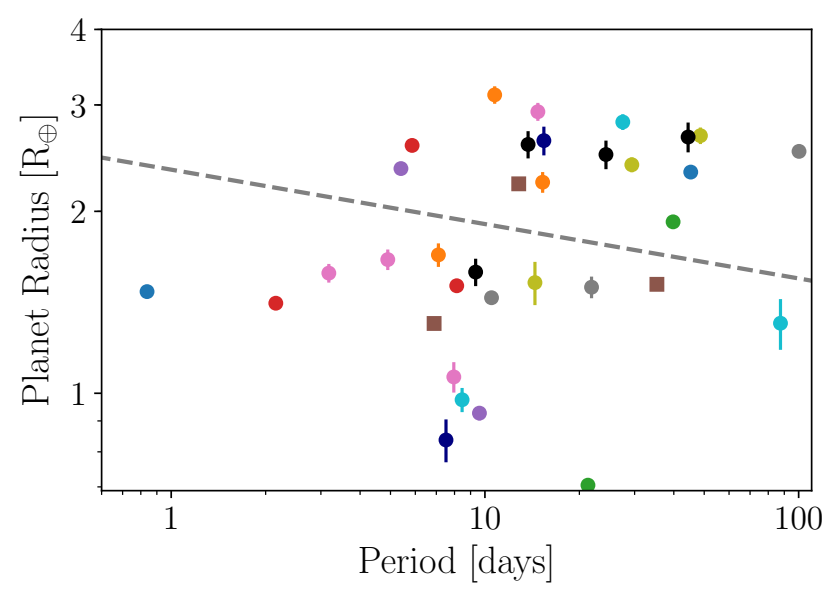

Figure 5. The radii and periods of planets from the asteroseismic sample in multi-transiting systems that straddle the radius gap. These 12 systems are analysed in Section 4.2 to derive the minimum masses for all the planets above the radius gap. Planets with the same colour indicate they are in the same system. Planets without visible error bars have errors smaller than the size of the symbols. The Kepler-100 system, which is found to be inconsistent with photoevaporation, is shown as square symbols rather than filled circles. The radius-gap derived by Van Eylen et al. (2018) is shown as the dashed line.

with any mass-constraint, an estimated RV semi-amplitude for the minimum mass (assuming a circular orbit), $K_{\text {cir }}$, and which super-Earth planet in the system these masses have been scaled from are listed in Table 1. As expected from our earlier discussion those systems which are most constraining have a either a large and hence massive planet below the gap, or a super-Earth exterior to the mini-Neptune, or both. Of those planets without mass constraints Kepler-130c is the standout case for follow-up with a minimum mass of $8.56 \mathrm{M}_{\oplus}$ in order to be consistent with photoevaporation. The system contains three detected transiting planets, the middle of which is a mini-Neptune while the other two are super-Earths with the longest period planet being the longest period super-Earth in the asteroseismic sample (the cyan points in Figure 5). Thus, with Kepler-130d a potentially long-period stripped rocky core, this system is a potentially unique laboratory for the potency of photoevaporation at long periods.

\subsubsection{Comparison to measured masses}

Of the nine planets with measured mass-constraints seven are clearly consistent, and Kepler-65c is consistent within $1 \sigma$. However, no solution can be found for the Kepler $-100 \mathrm{c} / \mathrm{d}$ planets at the $>3 \sigma$ level that is consistent with the photoevaporation model, this is discussed further in Section 5.1.

\subsection{CKS sample}

The CKS sample of planets by Petigura et al. (2017); Johnson et al. (2017) contains 457 multi-planet systems (Weiss et al. 2018a), 190 of which contain planets that straddle the gap with their mean radii (which we fix to occur at 
Owen, J. E. E Campos Estrada, B.

\begin{tabular}{llcccccc}
\hline Kepler-id & KOI & $\begin{array}{c}\text { Minimum } \\
\text { Mass }\left[\mathrm{M}_{\oplus}\right]\end{array}$ & $K_{\text {cir }}[\mathrm{m} / \mathrm{s}]$ & Measured Mass $\left[\mathrm{M}_{\oplus}\right]$ & Ref & Rocky Planet & $\begin{array}{c}\text { Rocky } \\
\text { Planet Mass }\left[\mathrm{M}_{\oplus}\right]\end{array}$ \\
\hline $100 \mathrm{c}$ & 41.01 & No Solution & - & $<7.05$ & 3 & $\mathrm{~d}$ & $4.94 \pm 0.45$ \\
$130 \mathrm{c}$ & 282.01 & 8.56 & 1.91 & - & - & $\mathrm{d}$ & $2.87 \pm 1.06$ \\
$65 \mathrm{c}$ & 85.01 & 6.31 & 1.99 & $5.4 \pm 1.7$ & 4 & $\mathrm{~d}$ & $4.8 \pm 0.51$ \\
$23 \mathrm{c}$ & 168.01 & 4.16 & 1.14 & $60.2_{-10.4}^{+11.4}$ & 2 & $\mathrm{~b}$ & $8.12 \pm 1.64$ \\
$23 \mathrm{~d}$ & 168.02 & 2.09 & 0.51 & $17.6_{-11.9}^{+13.7}$ & 2 & $\mathrm{~b}$ & $8.12 \pm 1.64$ \\
$338 \mathrm{~b}$ & 1930.01 & 2.64 & 0.65 & $30.6_{-21.1}^{+24.2}$ & 2 & $\mathrm{e}$ & $6.12 \pm 1.41$ \\
$338 \mathrm{c}$ & 1930.02 & 1.35 & 0.27 & - & - & $\mathrm{e}$ & $6.12 \pm 1.41$ \\
$338 \mathrm{~d}$ & 1930.03 & 0.78 & 0.13 & - & - & $\mathrm{e}$ & $6.12 \pm 1.41$ \\
$107 \mathrm{e}$ & 117.01 & 1.65 & 0.40 & $8.60 \pm 3.60$ & 5 & $\mathrm{c}$ & $7.41 \pm 1.25$ \\
$68 \mathrm{~b}$ & 246.01 & 1.41 & 0.50 & $7.65_{-1.32}^{+1.37}$ & 4 & $\mathrm{c}$ & $0.77 \pm 0.07$ \\
$127 \mathrm{c}$ & 271.02 & 1.18 & 0.21 & - & - & $\mathrm{b}$ & $5.32 \pm 1.81$ \\
$127 \mathrm{~d}$ & 271.01 & 0.77 & 0.12 & - & - & $\mathrm{b}$ & $5.32 \pm 1.81$ \\
$126 \mathrm{~d}$ & 260.02 & 0.66 & 0.08 & - & $\mathrm{c}$ & $4.75 \pm 0.81$ \\
$450 \mathrm{c}$ & 279.02 & 0.36 & 0.08 & - & - & $\mathrm{d}$ & $0.56 \pm 0.15$ \\
$37 \mathrm{~d}$ & 245.01 & 0.16 & 0.04 & $<12.2$ & 3 & $\mathrm{c}$ & $0.31 \pm 0.02$ \\
$10 \mathrm{c}$ & 72.02 & $<0.1$ & $<0.02$ & $7.37_{-1.19}^{+1.32}$ & 1 & $\mathrm{~b}$ & $4.38 \pm 0.31$ \\
\hline
\end{tabular}

Table 1. Predicted masses for planets in systems from the asteroseismic sample; systems are listed in descending order of predicted minimum mass. References for the measured masses: 1 - Rajpaul et al. (2017), 2 - Hadden \& Lithwick (2014), 3 - Marcy et al. (2014), 4 - Mills et al. (2019), 5 - Bonomo et al. (2019)

$1.85 \mathrm{R}_{\oplus}$, independent of period, for the CKS sample) and have mini-Neptunes with radius of $<6 \mathrm{R}_{\oplus}$. Only 63 of these systems contain planets which straddle the gap within $2 \sigma$ when accounting for radius errors, of which 2 were already analysed in the asteroseismic sample ${ }^{5}$. This leaves us 88 mini-Neptunes to perform our analysis on. The results of our analysis are shown in Table 2.

\subsubsection{Comparison to measured masses}

Of the 88 planets analysed in the CKS sample, 27 have some constraint on their masses. All planets are consistent with their measured masses; however the case of Kepler-172c/e system is worth noting. Only $\sim 1 \%$ of our Monte-Carlo samples for Kepler-172c produce a solution, the other $\sim 99 \%$ yield no solution in which both planets $\mathrm{c}$ and e are consistent with photoevaporation. However, all of the samples that are consistent with photoevaporation of the c/e system produce minimum masses that are consistent with the (weak) mass constraint for Kepler-172c. Having analysed > 100 planets in total it is not too surprising there is a planet that is only consistent at the $\sim 1 \%$ level. Thus, the Kepler- 172 system would benefit from further observations (either in the refinement of the planetary radii and/or masses).

Furthermore, we did analyse the Kepler-416 system ${ }^{6}$, this contains a candidate super-Earth - KOI1860.04 - with a radius of $\sim 1.5 R_{\oplus}$ and period 24.8 days Johnson et al. (2017). This would make it the longest period planet in the Kepler-416 system and as such the hardest super-Earth to completely strip. This would require the planet Kepler-416b, which has a period of $\sim 6.3$ days to have a minimum mass of $\sim 140 \mathrm{M}_{\oplus}$ in order to be consistent with photoevaporation. However, the planet candidate KOI1860.04 was sub-

5 Although the asteroseismic sample of systems is contained in the CKS sample, they do not all contain precise enough planetary radii to pass our $2 \sigma$ test.

6 This is not included in our 88 planets discussed above. sequently determined to have a false positive probability of $71 \%^{7}$. Removing this planet candidate from the system and analysing Kepler-416b again indicates it would be consistent with the photoevaporation model provided it had a mass $\gtrsim 1 \mathrm{M}_{\oplus}$.

Finally, the Kepler-142c/d system yields no solution at the $>3 \sigma$ level. Several of the Monte-Carlo samples do yield solutions, but with minimum masses of $\sim 30-40 \mathrm{M}_{\oplus}$ making it a nearly solid-core with $<0.1 \% \mathrm{H} / \mathrm{He}$ envelope which also seems unlikely. Therefore, like Kepler-100, we identify the Kepler-142 as being inconsistent with the photoevaporation model, this is discussed further in section 5.1.

\section{DISCUSSION}

Our results indicate that the photoevaporation model is in excellent agreement with the majority of observed multiplanet systems. We find 71 out of 73 systems and 35 out of the 36 planets with mass constraints are consistent with the photoevaporation model, despite 16 of those systems containing a super-Earth that is exterior to a mini-Neptune. Thus our first pass at testing the photoevaporation model with a large number of exoplanets indicates that they are consistent with the hypothesis that photoevaporation created the radius-gap, and the assumptions inferred from the photoevaporation model discussed in Section 2.1.

\subsection{Inconsistent Systems}

However, two out of the 73 systems (Kepler-100 and Kepler142) contained a mini-Neptune that could not retain its $\mathrm{H} / \mathrm{He}$ envelope if the longest period super-Earth had lost one

7 Obtained from the DR24 Kepler Reliability Report, downloaded from the NASA Exoplanet Archive on 29th August 2019 


\begin{tabular}{llcccccc}
\hline Kepler-id & KOI & $\begin{array}{c}\text { Minimum } \\
\text { Mass }\left[\mathrm{M}_{\oplus}\right]\end{array}$ & $K_{\text {cir }}[\mathrm{m} / \mathrm{s}]$ & Measured Mass $\left[\mathrm{M}_{\oplus}\right]$ & Ref & Rocky Planet & $\begin{array}{c}\text { Rocky } \\
\text { Planet Mass }\left[\mathrm{M}_{\oplus}\right]\end{array}$ \\
\hline $142 \mathrm{c}$ & 343.01 & No Solution & - & - & - & $\mathrm{d}$ & $2.86 \pm 1.00$ \\
$176 \mathrm{c}$ & 520.01 & $26.56^{*}$ & 8.16 & $23.0_{-8.0}^{+13.5}$ & 1 & $\mathrm{e}$ & $4.01 \pm 1.13$ \\
$176 \mathrm{~d}$ & 520.03 & 7.51 & 1.83 & $15.2_{-5.8}^{+10.4}$ & 1 & $\mathrm{e}$ & $4.01 \pm 1.13$ \\
$656 \mathrm{~b}$ & 732.01 & 13.26 & 8.44 & - & - & 732.03 & $2.62 \pm 0.70$ \\
$191 \mathrm{~d}$ & 582.01 & 4.70 & 1.82 & - & - & $\mathrm{b}$ & $3.59 \pm 0.95$ \\
$101 \mathrm{~b}$ & 46.01 & 4.61 & 1.77 & $51.1_{-4.7}^{+5.1}$ & 2 & $\mathrm{c}$ & $1.94 \pm 0.75$ \\
$105 \mathrm{~b}$ & 115.01 & 4.46 & 1.61 & $5.1_{-4.1}^{+6.3}$ & 1 & $\mathrm{c}$ & $4.87 \pm 1.33$ \\
$226 \mathrm{c}$ & 749.01 & 4.16 & 1.65 & $45.2_{-19.1}^{+2.5}$ & 1 & $\mathrm{~d}$ & $3.01 \pm 0.81$ \\
$307 \mathrm{~b}$ & 1576.01 & 3.50 & 1.05 & $8.8 \pm 0.9$ & 3 & 1576.03 & $1.79 \pm 0.73$ \\
$307 \mathrm{c}$ & 1576.02 & 2.53 & 0.70 & $3.9 \pm 0.7$ & 3 & 1576.03 & $1.79 \pm 0.73$ \\
$20 \mathrm{c}$ & 70.01 & 2.39 & 0.72 & $12.75_{-2.24}^{+2.17}$ & 4 & $\mathrm{f}$ & $1.08 \pm 0.24$ \\
$20 \mathrm{~d}$ & 70.03 & 0.14 & 0.02 & $10.07_{-3.70}^{+3.97}$ & 4 & $\mathrm{f}$ & $1.08 \pm 0.24$ \\
\hline & 102.01 & 2.21 & 1.14 & - & - & 102.02 & $1.21 \pm 0.37$ \\
$324 \mathrm{c}$ & 1831.01 & 1.81 & 0.34 & - & - & 1831.03 & $2.24 \pm 0.64$ \\
$102 \mathrm{e}$ & 82.01 & 1.71 & 0.5 & $8.93 \pm 2.0$ & 5 & $\mathrm{~d}$ & $3.96 \pm 1.22$ \\
- & 1276.01 & 1.70 & 0.41 & - & - & 1276.02 & $4.51 \pm 1.36$ \\
$282 \mathrm{~d}$ & 1278.01 & 1.60 & 0.38 & $61.0_{-36.1}^{+35.9}$ & 6 & $\mathrm{c}$ & $4.44 \pm 1.51$ \\
$282 \mathrm{e}$ & 1278.02 & 1.08 & 0.21 & $56.2_{-16.7}^{+16.2}$ & 6 & $\mathrm{c}$ & $4.44 \pm 1.51$ \\
$106 \mathrm{e}$ & 116.02 & 0.53 & 0.1 & $11.17 \pm 5.8$ & 5 & $\mathrm{~d}$ & $1.16 \pm 0.33$ \\
$106 \mathrm{c}$ & 116.01 & 1.59 & 0.43 & $10.44 \pm 3.2$ & 5 & $\mathrm{~d}$ & $1.16 \pm 0.33$ \\
$189 \mathrm{c}$ & 574.01 & 1.47 & 0.40 & $22.7_{-10.6}^{+17.1}$ & 1 & $\mathrm{~b}$ & $3.44 \pm 0.89$ \\
$173 \mathrm{c}$ & 511.01 & 1.47 & 0.45 & - & - & $\mathrm{b}$ & $4.61 \pm 1.21$ \\
\hline
\end{tabular}

Table 2. Those systems from the CKS sample which contain planets with predicted minimum masses $>1.4 \mathrm{M}_{\oplus}$, systems are listed in descending order of predicted minimum mass. The full table is available online. References for the measured masses: 1 - Hadden \& Lithwick (2014), 2 - Bonomo et al. (2014), 3 - Hadden \& Lithwick (2017), 4 - Buchhave et al. (2016), 5 - Marcy et al. (2014), 6 - Xie (2014), Notes: * $-0.5 \%$ upper-limit. The complete table is available online.

due to photoevaporation. These inconsistencies are demonstrated in Figure 6 for Kepler-100 and Figure 7 for Kepler142 , where we plot the mass-loss timescales for the superEarth and mini-Neptune as a function of the mini-Neptune's core mass (i.e. a version of the right-panel of Figure 1). These plots show that the Kepler-100 system is clearly inconsistent and that the Kepler-142 system is likely to be inconsistent (note the curves overlap, but only at high-masses $\left(\gtrsim 30 \mathrm{M}_{\oplus}\right)$, and this only occurs $<0.1 \%$ of the time, as discussed previously).

However, what is striking is that these two systems have a fairly similar architecture, as shown in Figure 8. Both systems contain three transiting planets, with the middle planet being above the radius gap, while the other two are below. What is also similar is that the period ratio between the interior two planets is $\sim 2$ (but not close to the 2:1 mean-motion resonance), while the period ratio between the exterior planets is much larger. Therefore, the dissimilar sizes and period ratios make these two systems rather unlike the standard Kepler multi-planet systems which have similar intra-system period and radius ratios (Weiss et al. 2018a,b).

Experimentation with changing the representative timescale for evaporation between for a wide range of plausible (and implausible) values does not bring these systems into agreement, neither does changing the core-composition if one requires it to be identical in both planets.

A trivial solution would be that the mass-loss efficiency is wrong. To bring these systems in line would require that the efficiency is underestimated for the outer planet relative to the planet above the gap (to allow the outer planets to lose their atmospheres faster). Such a solution seems unlikely as the outer planets are fairly low mass where the mass- loss efficiencies are already high. Further, these outer planets are at quite long orbital periods, where they might be close to the point of transitioning from hydrodynamic to Jeans escape (Owen \& Jackson 2012). Therefore, it is more likely the mass-loss efficiency for these planets is overestimated, rather than underestimated.

However, a solution can be found if the corecomposition of the mini-Neptune remains Earth-like, while the core density of the exterior super-Earth is lowered (making it easier to strip). Only a small increase in the core density (from $1 / 3$ iron to $\lesssim 5 \%$ iron) for Kepler-142d is required to make the system consistent. Though, for the Kepler-100 system, planet d requires a core-composition that is $\gtrsim 25 \%$ ice/water. Such dissimilar core densities are proposed in some formation scenarios (e.g. Raymond et al. 2018). There is some evidence that this could be the solution, as the measured mass-limit (95\% upper-limit) for Kepler-100d is $3 \mathrm{M}_{\oplus}$ (Marcy et al. 2014), significantly smaller than the assumed mass in our analysis, which was based on its radius and adopting an Earth-like composition of $4.95 \pm 0.45 \mathrm{M}_{\oplus}$ (see Table 1). To satisfy the mass upper-limit if the planet contains no $\mathrm{H} / \mathrm{He}$ atmosphere requires Kepler-100d to contain some water or ice. Therefore, it is plausible that the outer planets in these two systems having water/ice rich compositions can provide the solution to why they appear inconsistent with the photoevaporation model. We do however caution that the mass-limits of Kepler-100d assume a circular orbit (Marcy et al. 2014); however, it is now known from the asteroseismic analysis that it has a significant eccentricity of $0.38_{-0.16}^{0.12}$ (Van Eylen \& Albrecht 2015). Therefore it still remains open whether Kepler-100d could be ice/water rich or not. 


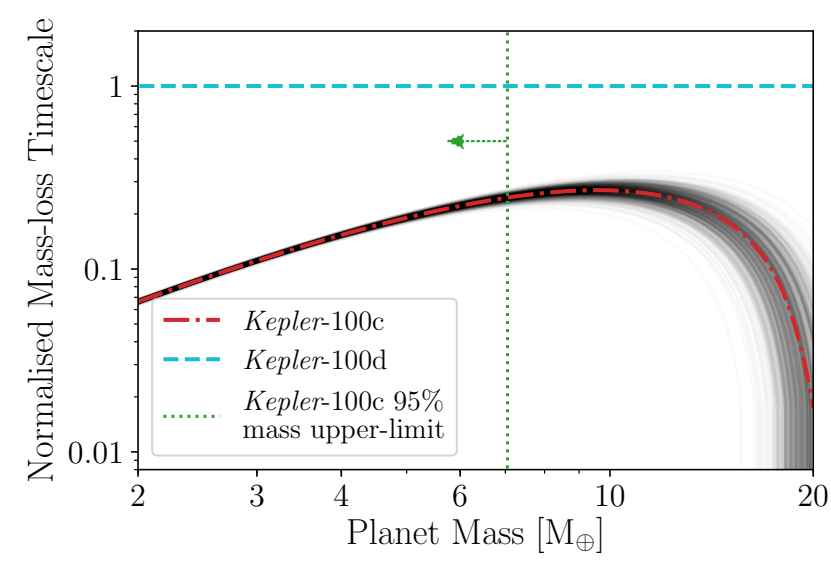

Figure 6. The mass-loss timescales as a function of core-mass for Kepler-100c \& d (normalised to the mean value for Kepler100d). The dot-dashed and dashed lines show the mean values and the translucent lines show 500 random draws. The dottedline indicates the 95\% upper-mass limit for Kepler-100c (Marcy et al. 2014). This Figure is similar to the overview example shown in Figure 1.

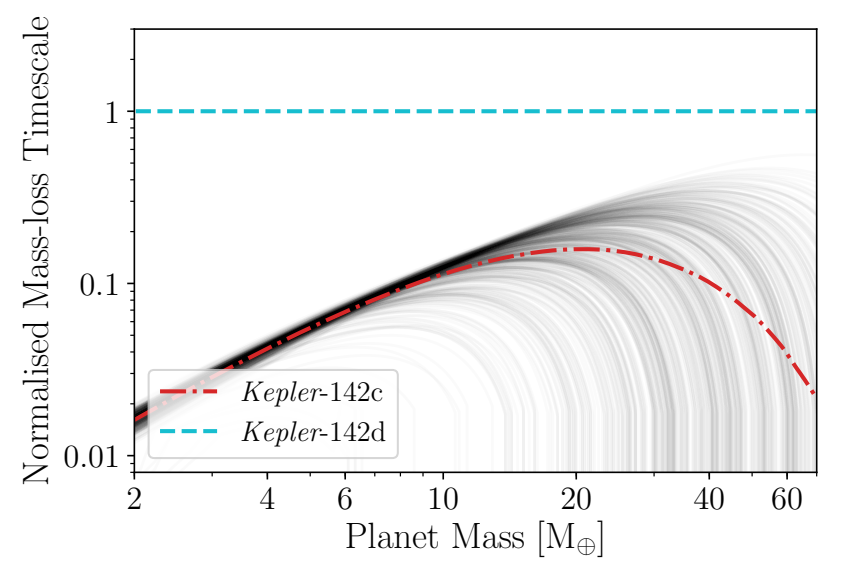

Figure 7. Same as above but for the Kepler-142c \& d system.

The fact that Kepler-100d is eccentric and at a significantly larger period ratio could point to another possible scenario: a giant-impact occurred after disc dispersal (e.g. Inamdar \& Schlichting 2016). Giant-impacts are efficient at removing $\mathrm{H} / \mathrm{He}$ atmospheres, as well as enhancing the affect of photoevaporation (e.g. Biersteker \& Schlichting 2019). There appears to be no eccentricity or mass measurement available for Kepler-142d; however, the similar orbital architecture indicates that this is also a plausible scenario for the Kepler-142 system.

Alternatively, the outer planets could have formed after disc dispersal in these two systems, never accreting an initial $\mathrm{H} / \mathrm{He}$ envelope or these two systems are indicating the photoevaporative efficiency scaling is incorrect, meaning these two planets still have $\mathrm{H} / \mathrm{He}$ atmospheres.

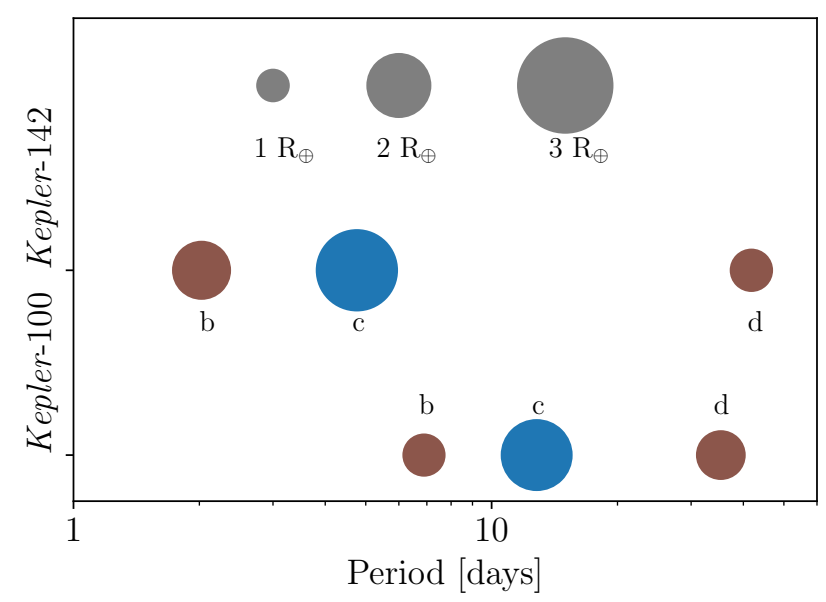

Figure 8. The architecture of Kepler-100 \& 142 systems shown in terms of the size and orbital period of their planets. Those planets identified as lying below the gap (and hence termed super-Earths in this work) are shown in brown and those identified as lying above the gap (hence termed mini-Neptunes) are shown in blue.

\section{$5.2 \quad$ Future comparisons}

We have identified two systems that are inconsistent with photoevaporation (Kepler-100 \& 142); however, further follow-up of these systems would illuminate as to the origin of this disagreement: dissimilar core densities, giant-impacts or something else. For Kepler-100, refitting the masses assuming a non-circular orbit is a priority. For Kepler-142, using the precise constraints on the stellar density from the CKS survey and GAIA (e.g. Petigura et al. 2017; Fulton \& Petigura 2018) to refit the transit photometry would improve radii and eccentricity estimates, as well as obtaining follow-up RV masses. Further, observing the system with $C H E O P S$ to obtain precise radii estimates would also improve the constraints. Kepler-176 is also a system worth investigating in greater detail: as well as refitting the transit photometry or observing the system with CHEOPS, improving the transit-timing mass-measurements once the Kepler field is observed by TESS will indicate whether this system is consistent with the photoevaporation model or not. Further, we note the predicted masses of Kepler-176c \& d imply they are separated by $\sim 10$ mutual hill radii, implying they may be close to dynamical stability.

Furthermore, we have provided a list of 12 planets which have estimated RV semi-amplitudes $>1 \mathrm{~m} / \mathrm{s}$ which should be prime targets for spectroscopic follow-up observations. Our method should be directly applicable to multi-planet systems that TESS finds and where RV follow-up is easier. In anticipation of such possibilities the software used to estimate the minimum masses is available to the public. We also note that using RV follow-up to test the photoevaporation model may require going beyond $3 \sigma$ mass measurements, and that upper-limits can be equally useful ${ }^{8}$.

8 We suspect there may even be RV data already available to test some of the Kepler-systems in Tables $1 \& 2$ that is yet to be published as a $3 \sigma$ mass detection. 


\section{SUMMARY}

We have shown how the photoevaporation model can be used to estimate the minimum masses of planets that are hypothesised to posses large hydrogen/helium atmospheres if they reside in a system that also contains a planet below the exoplanet radius-gap. Our approach is valuable as it is independent of the host star's uncertain EUV/X-ray history. This method essentially answers the following question: given photoevaporation had to completely strip the planet that resides below the radius-gap, how massive does the planet above the radius-gap need to be to retain its hydrogen/helium envelope? Unsurprisingly, the most stringent constraints come from systems in which the planet below the gap is large, and is exterior to the planet above the gap.

We have applied this method to 104 exoplanets that likely reside above the radius gap (within $2 \sigma$ ) in 73 systems that likely contain a planet that resides below the radiusgap (within $2 \sigma$ ). In general, we find excellent agreement between the photoevaporation model and those planets with measured mass constraints, indicating that photoevaporation is the cause of the radius-gap. Only two planets (Kepler $100 \mathrm{c}$ and 142c) are inconsistent with the photoevaporation at the $>3 \sigma$ level; and we speculate that these systems could either contain planets with dissimilar core compositions, underwent a giant impact, had planets that both formed before and after the gas disc dispersed, or simply the mass-loss rates are incorrect. Any of these hypothesis make these systems unusual in terms of their evolutionary pathway when compared to the bulk of the population, and warrant more detailed study.

We have identified 12 planets with RV semi-amplitudes $\gtrsim 1 \mathrm{~m} \mathrm{~s}^{-1}$, the vast majority of which only have weak TTV mass constraints or no measured mass. Therefore, these planets would be prime targets for testing the photoevaporation model either with RV follow-up or additional studies of TTVs when TESS observes the Kepler field.

Finally, our method can be applied to any multi-planet systems that contain planets which straddle the radius-gap. Therefore, ongoing and future transit surveys should provide a detailed test of the photoevaporation model, provided they are combined with a well designed follow-up program to measure masses. We emphasis that upper-mass limits are equally useful as mass measurements, since our method predicts a minimum planet mass. We also suspect this method of comparing planets in the same system could be applied to other mass-loss mechanisms e.g. core-powered mass-loss (Ginzburg et al. 2018)

\section{ACKNOWLEDGEMENTS}

We thank the referee for a report which improved the manuscript. JEO is supported by a Royal Society University Research Fellowship. We are grateful to Vincent van Eylen, the CKS team and the Kepler team for making their work publicly available to allow us to perform this analysis. This research has made use of the NASA Exoplanet Archive, which is operated by the California Institute of Technology, under contract with the National Aeronautics and Space Administration under the Exoplanet Exploration Program. This work was performed using the DiRAC Data
Intensive service at Leicester, operated by the University of Leicester IT Services, which forms part of the STFC DiRAC HPC Facility (www.dirac.ac.uk). The equipment was funded by BEIS capital funding via STFC capital grants ST/K000373/1 and ST/R002363/1 and STFC DiRAC Operations grant ST/R001014/1. DiRAC is part of the National e-Infrastructure.

\section{REFERENCES}

Allart R., et al., 2018, Science, 362, 1384

Baraffe I., Chabrier G., Barman T. S., Selsis F., Allard F., Hauschildt P. H., 2005, A\&A, 436, L47

Barclay T., Pepper J., Quintana E. V., 2018, ApJS, 239, 2

Biersteker J. B., Schlichting H. E., 2019, MNRAS, 485, 4454

Bonomo A. S., et al., 2014, A\&A, 572, A2

Bonomo A. S., et al., 2019, Nature Astronomy, 3, 416

Borucki W. J., et al., 2011, ApJ, 736, 19

Buchhave L. A., et al., 2016, AJ, 152, 160

Carter J. A., et al., 2012, Science, 337, 556

Chen H., Rogers L. A., 2016, ApJ, 831, 180

Dorn C., Harrison J. H. D., Bonsor A., Hands T. O., 2019, MNRAS, 484, 712

Dressing C. D., et al., 2015, ApJ, 800, 135

Ehrenreich D., et al., 2015, Nature, 522, 459

Fortney J. J., Marley M. S., Barnes J. W., 2007, ApJ, 659, 1661

Fressin F., et al., 2013, ApJ, 766, 81

Fulton B. J., Petigura E. A., 2018, AJ, 156, 264

Fulton B. J., et al., 2017, AJ, 154, 109

Ginzburg S., Schlichting H. E., Sari R., 2018, MNRAS, 476, 759

Günther M. N., Queloz D., Demory B.-O., Bouchy F., 2017, MNRAS, 465, 3379

Gupta A., Schlichting H. E., 2019a, arXiv e-prints, p. arXiv:1907.03732

Gupta A., Schlichting H. E., 2019b, MNRAS, 487, 24

Hadden S., Lithwick Y., 2014, ApJ, 787, 80

Hadden S., Lithwick Y., 2017, AJ, 154, 5

Howard A. W., et al., 2010, Science, 330, 653

Inamdar N. K., Schlichting H. E., 2016, ApJ, 817, L13

Jankovic M. R., Owen J. E., Mohanty S., 2019, MNRAS, 484, 2296

Jin S., Mordasini C., 2018, ApJ, 853, 163

Jin S., Mordasini C., Parmentier V., van Boekel R., Henning T., Ji J., 2014, ApJ, 795, 65

Johnson J. A., et al., 2017, The Astronomical Journal, 154, 108

Jontof-Hutter D., et al., 2016, ApJ, 820, 39

Kubyshkina D., et al., 2018, A\&A, 619, A151

Lammer H., Selsis F., Ribas I., Guinan E. F., Bauer S. J., Weiss W. W., 2003, ApJ, 598, L121

Lecavelier Des Etangs A., et al., 2010, A\&A, 514, A72

Lopez E. D., Fortney J. J., 2013, ApJ, 776, 2

Lopez E. D., Fortney J. J., Miller N., 2012, ApJ, 761, 59

Marcy G. W., et al., 2014, ApJS, 210, 20

Mayor M., et al., 2011, arXiv e-prints, p. arXiv:1109.2497

Mills S. M., et al., 2019, The Astronomical Journal, 157, 145

Mulders G. D., Pascucci I., Apai D., Ciesla F. J., 2018, AJ, 156, 24

Murray-Clay R. A., Chiang E. I., Murray N., 2009, ApJ, 693, 23

Owen J. E., 2019, Annual Review of Earth and Planetary Sciences, 47, 67

Owen J. E., Adams F. C., 2019, MNRAS submitted,

Owen J. E., Jackson A. P., 2012, MNRAS, 425, 2931

Owen J. E., Morton T. D., 2016, ApJ, 819, L10

Owen J. E., Wu Y., 2013, ApJ, 775, 105

Owen J. E., Wu Y., 2017, ApJ, 847, 29 
Paxton B., Bildsten L., Dotter A., Herwig F., Lesaffre P., Timmes F., 2011, ApJS, 192, 3

Paxton B., et al., 2013, ApJS, 208, 4

Petigura E. A., et al., 2017, The Astronomical Journal, 154, 107

Rajpaul V., Buchhave L. A., Aigrain S., 2017, Monthly Notices of the Royal Astronomical Society, 471, L125

Raymond S. N., Boulet T., Izidoro A., Esteves L., Bitsch B., 2018, MNRAS, 479, L81

Rogers L. A., 2015, ApJ, 801, 41

Rogers L. A., Seager S., 2010, ApJ, 712, 974

Silburt A., Gaidos E., Wu Y., 2015, ApJ, 799, 180

Spake J. J., et al., 2018, Nature, 557, 68

Thompson S. E., et al., 2018, ApJS, 235, 38

Tu L., Johnstone C. P., Güdel M., Lammer H., 2015, A\&A, 577, L3

Valencia D., Sasselov D. D., O'Connell R. J., 2007, ApJ, 665, 1413

Van Eylen V., Albrecht S., 2015, ApJ, 808, 126

Van Eylen V., Agentoft C., Lundkvist M. S., Kjeldsen H., Owen J. E., Fulton B. J., Petigura E., Snellen I., 2018, MNRAS, 479,4786

Vidal-Madjar A., Lecavelier des Etangs A., Désert J. M., Ballester G. E., Ferlet R., Hébrard G., Mayor M., 2003, Nature, 422, 143

Weiss L. M., Marcy G. W., 2014, ApJ, 783, L6

Weiss L. M., et al., 2018a, AJ, 155, 48

Weiss L. M., et al., 2018b, AJ, 156, 254

Wu Y., 2019, ApJ, 874, 91

Wu Y., Lithwick Y., 2013, ApJ, 772, 74

Wyatt M. C., Kral Q., Sinclair C. A., 2019, MNRAS, p. 2660

Xie J.-W., 2014, ApJS, 210, 25

Zeng L., et al., 2019, Proceedings of the National Academy of Sciences, 116, 9723

Zink J. K., Christiansen J. L., Hansen B. M. S., 2019, MNRAS, 483, 4479

This paper has been typeset from a $\mathrm{T}_{\mathrm{EX}} \mathrm{L} \mathrm{LT}_{\mathrm{E}} \mathrm{X}$ file prepared by the author. 